\title{
A STEP CYCLE SLIP DETECTION AND REPAIR METHOD BASED ON DOUBLE- CONSTRAINT OF EPHEMERIS AND SMOOTHED PSEUDORANGE
}

\author{
Fangchao LI ${ }^{1,2)}$, Jingxiang GAO ${ }^{1,2) *}$, Zengke LI ${ }^{1,2)}$, Nijia QIAN ${ }^{1,2)}$, \\ Liu YANG ${ }^{1,2)}$ and Yifei YAO ${ }^{3}$ ) \\ 1) NASG Key Laboratory of Land Environment and Disaster Monitoring, China University of Mining and Technology, Xuzhou, China \\ ${ }^{2)}$ School of Environment Science and Spatial Informatics, China University of Mining and Technology, Xuzhou, China \\ ${ }^{3)}$ College of Water Resources and Architectural Engineering, Northwest A\&F University, Yangling, China
}

*Corresponding author's e-mail: jxgao@cumt.edu.cn

\section{ARTICLE INFO}

Article history

Received 15 April 2019

Accepted 20 August 2019

Available online 13 November 2019

\section{Keywords:}

Satellite orbit

Cycle slip detection and repair

Smoothed pseudorange

Linear combination

Ionospheric variation

\begin{abstract}
Most cycle slip (CS) processing methods are based on the linear combinations of pseudorange and phase observation, and the gross error on pseudorange can lead to mistakes on the results. From this perspective, a novel method is proposed relying on satellite orbit and smoothed pseudorange, which is divided into two steps: rough and detailed process. During the first process, we calculate and compare the TD (Time Difference) model difference between satelliteto-receiver distance and phase observation to get the systematic error. We use the difference between the predicted and calculated to detect and fix the massive mistake. During the second process, we add -3 to +3 cycles on phase observation which is used to smooth pseudorange by an eliminating ionospheric method, then we search the unique error by validating the ambiguity of WL-MW, L1 and L2, and the difference of smoothed pseudorange and phase observation. Besides, the detectors constructed by ionospheric information of previous epochs are used to test the estimation by LS (least square). The first process can correctly detect and repair the integer error for high sampling rate datasets while limiting it in a small range for low sampling rate datasets. The datasets from IGS (International GNSS Service) with low and high sampling rate are used to verify the proposed method. High sampling rate datasets experiment shows there are only 4 among 640.778 observations from GODN is failed to repair the error when testing 9 stations. Low sampling rate datasets experiment shows that the average success rate of all stations is about $99.56 \%$. The lowest success rate of L1 and L2 frequency reaches $99.20 \%$ and $99.21 \%$, respectively of DYNG station, while the highest reaches $99.83 \%$ of both L1 and L2 of HRAO station.
\end{abstract}

\section{INTRODUCTION}

Phase observations are widely used in positioning and navigation for its high-precision, and the continuous tracking signals from satellites can ensure the continuity of integer ambiguity. The phase observation error whose integral part is wrong while the fractional part is correct is called CS (cycle slip), which is caused by reinitializing the integer counter when losing lock GNSS signal. If the error occurs and not repairs correctly, it will directly have an unknown integer cycles biases on the integer ambiguity and a deviation on positioning result.

In the past fewer decades, significant achievement has obtained to solve the particular error, and we classify the methods into three categories: linear combination, Bayesian theory, and the assistant algorithm by inertial technology. The first classical method is linear combinations, which is widely used for its simplicity and convenience, such as GF (geometry-free), HMW (Hatch- Melbourne-Wübbena) combination (Blewitt, 1990; Hatch, 1982; Melbourne, 1985; Wübbena, 1985), and their related expanded forms (de Lacy et al., 2008). Liu proposed a new automated method, which considers the ionospheric variation and requires a high precision of pseudorange and only tested the high sampling rate data (Liu, 2011). Li et al. searched for optimal combination coefficients by pseudorange and phase observation ( $\mathrm{Li}$ et al., 2011). Huang et al. designed one GF combination and two pseudorange minus phase combinations and verified the algorithm with artificial integer error on measured data (Huang et al., 2012). De Lacy et al. organized five GF linear combinations, but it requires a high precision of pseudorange about $10-15 \mathrm{~cm}$, which is quite a difficult thing to be satisfied in real-world circumstances. Also, the algorithm only teste high sampling rate dataset (de Lacy et al., 2012). Considering the second-order ionospheric residual information, Cai et al. introduce an HMW linear combination. Unfortunately, it is not suitable for real-time observation (Cai et al., 2013). Zhang and $\mathrm{Li}$ introduce an extended TD model with triple-frequency signals (Zhang and Li, 2016), but it is only suitable for quiet ionospheric condition. Zhao et 
al. improved HMW combination and combined triplefrequency signals for the disturbed ionosphere situation (Zhao et al., 2019). However, a common feature of the linear combination is to weaken the ionospheric delay, which makes problems that there may be an insensitive error, and the frequency occurs $\mathrm{CS}$ is challenging to identify. Also, most of the above methods are not suitable for low sample rate data at the disturbed ionosphere condition, and the gross error on pseudorange can lead to mistakes in the result. The second category is the Bayesian theory, which is used to undifferenced cases (de Lacy, 2008). However, several continuous epoch data before and after the current epoch is required to satisfy some criteria (Zhang and Li, 2012). A good result can be obtained from least-squares adjustment, even if the sampling rate of the data reaches $30 \mathrm{~s}$, but it requires a high precision of the ionospheric delay information (Banville and Langley, 2013). Even if it has got the precise coordinate of satellite and station, the TD accuracy of non-dispersive noise is constrained in $0.02 \mathrm{~m}$ to strengthen LS adjustment mathematical model (Zhang and Li, 2016). Xiao et al. enhanced LS model with a separating method, but a clean data before is required (Xiao et al., 2018). However, the above techniques require clean data before or after the detect time. The third kind is aiding by INS (Inertial Navigation System) (Colombo et al., 1999). Altmayer establishes detectors by the standard deviation of the previous (Altmayer, 2000), while Lee et al. use the INS position (Lee et al., 2003), and Du and Gao constructed WL and extra WL by INS information (Du and Gao, 2012). Younsil et al. combined low cast MEMS (Micro Electro Mechanical Systems) INS and single-frequency differential GPS signal (Younsil et al., 2013). Li constructed three detectors using INS information (Li et al., 2016). However, the INS constraints the cost and feasibility of the hardware.

To satisfy real-time navigation and positioning, we present an effective and efficient algorithm based on double-constraint of ephemeris and the smoothed pseudorange, which is divided into two steps: rough and detailed process. During the first process, we obtain systematic error from the comparison of the TD model of geometric distance and phase observations. Then, the difference is compared to detect and repair massive error (such as more than three cycles). During the second process, the phase observation added -3 to 3 cycles error is used to smooth the pseudorange. Then we verify the ambiguity of MW-WL, L1 and L2, the residual of carrier phase and smoothed pseudorange. Finally, the ionospheric variation predicted and observed is used as a detector to identify and fix some particular error not handle during the previous process.

In the following sections, we introduce the mathematical model of satellite positioning firstly. Then the proposed algorithm is described with a flow chart and descriptive language. Next, the measured datasets from IGS (International GNSS Service) is an example to validate the algorithm, and we analyze reasons failing to execute the algorithm for some epochs. Finally, it made a summary and outlook.

\section{THE FUNDAMENTAL KNOWLEDGE}

To improve the success rate, we present the mathematic models of GPS, and its linear combinations, such as MW, WL combination firstly. Then, we smooth the pseudorange, considering the ionospheric variation in this section.

\subsection{MATHEMATIC MODELS OF GPS}

The un-differenced and un-combined mathematic models of pseudorange and phase observations are simplified as follows:

$$
\begin{aligned}
& P_{j}=\rho+c \delta \tau_{r}+c \delta \tau_{s}-u_{j} v+\varepsilon_{p} \\
& \lambda_{j} \varphi_{j}=\rho+c \delta \tau_{r}+c \delta \tau_{s}+\lambda_{j} z_{j}+u_{j} v+\varepsilon_{\varphi}
\end{aligned}
$$

Where subscript $j$ indicates the frequency. $\lambda$ indicates wavelength. $P$ is pseudorange with meters. $\varphi$ is phase observation with cycles. $\rho$ is the geometric distance between the phase center of the receiver and satellite antenna. $c$ is light velocity in vacuum. $\delta \tau_{r}$ and $\delta \tau_{s}$ indicate clock correction of receiver and satellite. $z$ is integer ambiguity. $u_{j}$ is the ionospheric coefficient of different frequencies with $u_{1}=1$ for L1, and $u_{2}=f_{1}^{2} / f_{2}^{2}$ for L2. $\varepsilon_{p}$ and $\varepsilon_{\varphi}$ indicate the measurement error of pseudorange and phase observation. We do not consider other errors since the TD model can eliminate them with a detail explication by (Hieu et al., 2014).

The $\Delta$ indicates the difference of adjacent epochs, and we make a TD model of phase observations as equation (3). If there exists error $\Delta z \neq 0$, nevertheless $\Delta z=0$.

$$
\lambda_{j} \Delta \varphi_{j}=\Delta \rho+c \Delta \delta \tau_{r}+c \Delta \delta \tau_{s}+\lambda_{j} \Delta z_{j}+u_{j} \Delta v+\varepsilon_{\Delta \varphi}
$$

For convenience, we simplify the models and only consider the ionospheric delay when deducting formula. Based on the simplified model, we express the WL combination of pseudorange and phase observations as follow (Blewitt, 1990):

$$
P_{\Delta}(t)=\left(f_{1} P_{1}(t)-f_{2} P_{2}(t)\right) /\left(f_{1}+f_{2}\right)=\rho(t)-u_{\Delta} v(t)+\varepsilon_{F}
$$

$\lambda_{\Delta} \varphi_{\Delta}(t)=\lambda_{\Delta}\left(\varphi_{1}(t)-\varphi_{2}(t)\right)=\rho(t)+\lambda_{\Delta} z_{\Delta}-u_{\Delta} v(t)+\varepsilon_{\varphi_{\Delta}}$

Where $P_{\Delta}$ indicates pseudorange with WL combination; $\lambda_{\Delta}$ is the wavelength whose value is $\lambda_{\Delta}=c /\left(f_{1}-f_{2}\right) \approx 86.19 \mathrm{~cm} ; \quad z_{\Delta}$ is the integer ambiguity whose value is $z_{\Delta}=z_{1}-z_{2} ; u_{\Delta}$ is ionospheric delay coefficient with a value $u_{\Delta}=-f_{1} / f_{2}$. 
Combining the equation (4) and (5) of L1 and L2 frequencies, we can express the $z_{\Delta}$ and $\Delta z_{\Delta}$ as follows:

$$
\begin{aligned}
& z_{\Delta}=\varphi_{\Delta}(t)-P_{\Delta}(t) / \lambda_{\Delta} \\
& \Delta z_{\Delta}=\Delta \varphi_{\Delta}(t)-\Delta P_{\Delta}(t) / \lambda_{\Delta}
\end{aligned}
$$

Additionally, MW-WL (Melbourne, 1985; Wübbena, 1985) combination is a useful model as follows:

$$
\left(z_{1}-z_{2}\right)=\frac{c\left(f_{1} P_{1}+f_{2} P_{2}\right)}{\left(f_{1}+f_{2}\right)\left(f_{1}-f_{2}\right)}-\left(\varphi_{1}-\varphi_{2}\right)
$$

From equation (8), the MW-WL combination eliminates lots of errors, such as the ionospheric delay, clock bias and of satellite and receiver, the geometric distance between satellites and receiver. Also, the MW-WL has quite a long-wavelength to detect and repair the unique error.

\subsection{CARRIER SMOOTHING PSEUDORANGE CONSIDERING IONOSPHERIC VARIATION}

The traditional pseudorange smoother, which believes that the ionospheric delay is a constant, is shown as follows:

$$
P_{s j}(t)=\frac{1}{\alpha} P_{j}(t)+\frac{\alpha-1}{\alpha}\left(P_{s j}(t-1)+\lambda_{j}\left(\varphi_{j}(t)-\varphi_{j}(t-1)\right)\right)
$$

Where $P_{s}$ is smoothed pseudorange, $\alpha$ is a smoothing coefficient.

We can calculate the smoothed pseudorange of the first epoch as follow:

$$
P_{s j}\left(t_{0}\right)=\frac{1}{K} \sum_{k=0}^{K}\left(P_{j}\left(t_{0}-k\right)+\lambda_{j}\left(\varphi_{j}\left(t_{0}\right)-\varphi_{j}\left(t_{0}-k\right)\right)\right)
$$

Where $K$ is the number of smooth epoch and $t_{0}$ is the time of first epoch.

The traditional smoother has two strikes against it. One is that the $P_{s}$ would absorb twice ionospheric variation if the filter runs for a long time. The other is that it needs a long time to weaken or eliminate the systematic error of first epoch. To extend the running time of the smoother, we use a new filter that can reduce the ionospheric variation to smooth pseudorange and explain it as below:

$$
\begin{aligned}
P_{s j}(t) & =\frac{1}{\alpha} P_{j}(t)+\frac{\alpha-1}{\alpha}\left(P_{s j}(t-1)+\lambda_{j}\left(\varphi_{j}(t)-\right.\right. \\
& \left.\left.-\varphi_{j}(t-1)\right)\right)-2 u_{j} \Delta v(t, t-1)
\end{aligned}
$$

The following equation can be used to initialize the $P_{s}^{\prime}\left(t_{0}\right)$ with several precise observation.

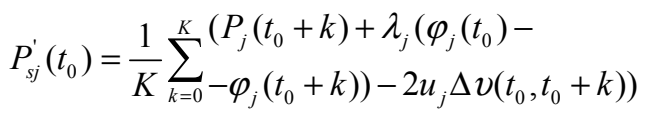

We obtain the ionosphere variation, $\Delta v(t, t+k)$, accurately from the following equation.

$$
\Delta v(t, t+k)=\left(\lambda_{i} \Delta \varphi_{i}(t, t+k)-\lambda_{j} \Delta \varphi_{j}(t, t+k)\right) /\left(u_{i}-u_{j}\right)
$$

\section{THE PROPOSED METHOD}

We divided the proposed method into two parts, rough and detailed progress, and we introduce the first progress in 3.1 section while the second in 3.2. In 3.3 section, we present the whole process by flow chart and descriptive language.

\subsection{ROUGH PART OF THE PROPOSED METHOD}

In this section, we will discuss how to detect and repair the massive error. Using the Taylor formula, we use the receiver position obtained from SPP (Standard Point Positioning) and satellite location provided by precise/broadcast ephemeris to express the satellite-toreceiver distance, which is as follows:

$$
\tilde{\rho}(t)=\rho_{0}(t)+G(t) \Delta X(t)+G(t) \Delta x(t)
$$

Where $G$ is the direction cosine matrix. $\rho_{0}$ indicates geometric distance calculated by approximate receiver position and satellite position. $\Delta X(t)$ and $\Delta x$ show position variation of receiver and satellite, respectively.

We present the TD of equation (14) as follows:

$$
\begin{aligned}
\Delta \tilde{\rho}(t, t-1) & =\Delta \rho_{0}(t, t-1)+G(t-1)(\Delta X(t)-\Delta X(t-1))+ \\
& +(G(t)-G(t-1)) \Delta X(t)+G(t-1)(\Delta x(t)-\Delta x(t-1 \\
& +(G(t)-G(t-1)) \Delta x(t)
\end{aligned}
$$

Next, we will mainly discuss the influence of satellite and receiver position, velocity on $\Delta \tilde{\rho}(t, t-1)$.

The $\zeta_{1}$ is defined as follows:

$$
\begin{aligned}
\zeta_{1} & =G(t-1)(\Delta X(t)-\Delta X(t-1)) \\
& =G(t-1)((\tilde{X}(t)-\tilde{X}(t-1))-(\bar{X}(t)-\bar{X}(t-1))) / \delta t \times \delta t \\
& =G(t-1)(\tilde{\omega}-\bar{\omega})=G(t-1) \times d \omega \times \delta t
\end{aligned}
$$

Where $\tilde{X}$ and $\tilde{\omega}$ indicate real satellite coordinates and velocity while $\bar{X}$ and $\bar{\omega}$ indicate satellite coordinates and velocity provided by ephemeris. $\delta t$ is sampling interval rate, and $d \omega$ is the error of satellite velocity.

Current research shows that the real error of satellite velocity provided by ephemeris is less than 1 $\mathrm{cm} / \mathrm{s}$ (Serrano et al., 2004), and the difference of satellite velocity calculated by navigation file and precise ephemeris is in $\mathrm{mm} / \mathrm{s}$ level. Thus, if we replace $\Delta \tilde{\rho}$ with $\Delta \rho_{0}$, the influence of $\zeta_{1}$ on $\Delta \tilde{\rho}$ is only several millimeters. 


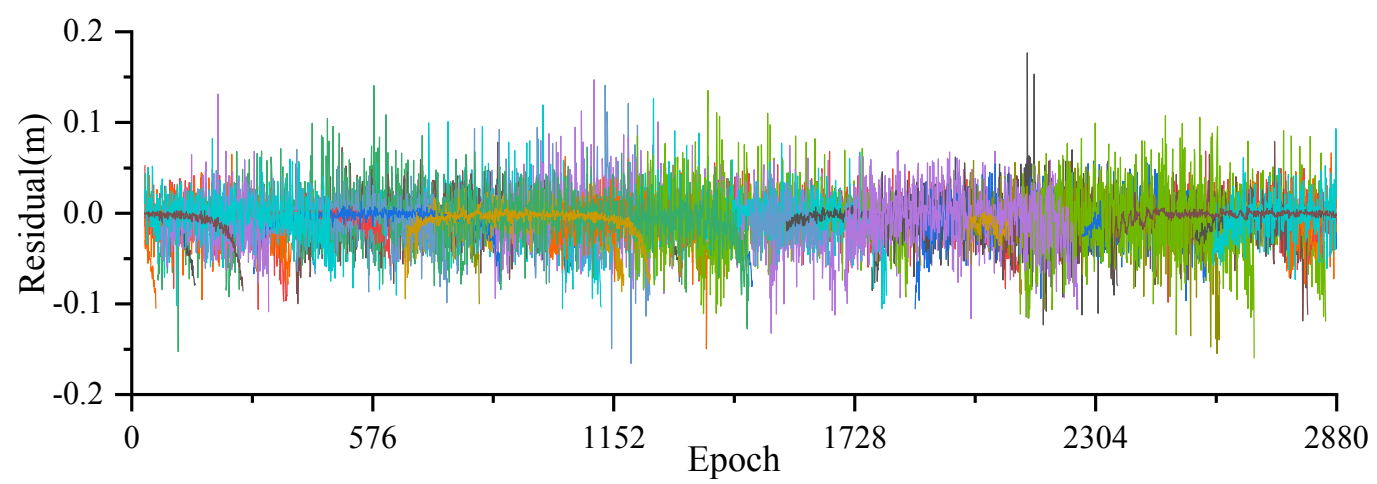

Fig. 1 The comparison of $\eta$ predicted and observed with $30 \mathrm{~s}$ sampling rate data.

Analogously, we define $\zeta_{2}$ as below:

$$
\zeta_{2}=(G(t)-G(t-1)) \Delta X(t)
$$

The research of Li et al. shows that $G(t)-G(t-1)$ is less than $5 \times 10^{-3}$ even if the sampling interval is the $60 \mathrm{~s}$ (Li et al., 2019), and it can be smaller if we provide a lower sampling interval rate. $\Delta X(t)$ is ephemeris error which is less than 1 meter. Thus, if we replace $\Delta \tilde{\rho}$ with $\Delta \rho_{0}$, the influence of $\zeta_{2}$ on $\Delta \tilde{\rho}$ is several millimeters, even if the sampling interval is the $60 \mathrm{~s}$.

The several epochs are used to estimate approximate receiver coordinate for a static receiver, and we can obtain equation as follows:

$\xi_{1}=G(t-1)(\Delta x(t)-\Delta x(t-1))=0$

Similarly, we define $\xi_{2}$ as follow:

$\xi_{2}=(G(t)-G(t-1)) \Delta x(t)$

The SPP can obtain a sub-meter level positioning accuracy and $G(t)-G(t-1)$ is less than $5 \times 10^{-3}$, and the $G(t)$ is direction cosines which is less than 1 . Thus, if we replace $\Delta \tilde{\rho}$ with $\Delta \rho_{0}$, the influence of $\xi_{2}$ on $\Delta \tilde{\rho}$ is less than $1 \mathrm{~cm}$.

In the above discussion, even if we replaced $\Delta \tilde{\rho}$ by $\Delta \rho_{0}$, the influence of $\zeta_{1}, \zeta_{2}, \xi_{1}$ and $\xi_{2}$ on $\Delta \tilde{\rho}(t, t-1)$ is only several centimeters. What is mean, there is little influence, if we take $\Delta \tilde{\rho}$ instead of $\Delta \rho_{0}$.

If we take equation (15) into equation (3), we can get the following equation:

$$
\lambda_{j} \Delta \varphi_{j}=\Delta \rho_{0}+c \Delta \delta \tau_{r}+c \Delta \delta \tau_{s}+\lambda_{j} \Delta z_{j}+u_{j} \Delta v+\varepsilon_{\Delta \varphi}
$$

We define $\eta=c \Delta \delta \tau_{r}+c \Delta \delta \tau_{s}+\lambda_{j} \Delta z_{j}+u_{j} \Delta v$, and limit the unique error at a range because we can predict $\eta$ with a sure accuracy using polynomial fitting. In order to investigate the prediction precision of $\eta$, the $30 \mathrm{~s}$ sampling rate dataset of GODN is an example to show the difference of $\eta$ predicted and observed in Figure 1, and different colors represent different satellites in latter similar figures.

The ionospheric activity has been considered as one of the factors to investigate the influence on $\eta$. The $K_{p}$ index provided by GFZ in Potsdam, Germany (http://www.gfz-potsdam.de/en/kp-index/) is used to select the observations since it can reflect the levels of the ionospheric activity by geomagnetic disturbances.

The real IGS GODN datasets measured on doy (day of the year) 291 (the quietest ionosphere activity day) and doy 238 (the most disturbing ionosphere activity day) of 2018 is an example to investigate the influence of ionosphere on $\eta$. We show the two results in Figure 2 and Figure 3, respectively.

From comparing Figure 2 and Figure 3, we can get that, the maximum difference of $\eta$ is less than $15 \mathrm{~cm}$, and the $\eta$ is higher at a disturbed ionospheric activity condition than a quiet condition. Furthermore, the ionospheric activity may influence the prediction of $\eta$, which may be more than one cycle and leading to a cycle deviation. However, the difference of $\eta$ by the predicted and observed is less than 3 cycles, and the first process can detect and repair the massive error, accurately.

The contrast of $\eta$ between the predicted and observed is only several decimeters for GODN station, and it is easy to detect the massive error. Moreover, we can repair it according to the following formula by comparing $\eta$ by predicted and observed.

$\Delta z_{j}(t)=H_{\text {round }} \frac{H(\eta(t-1), \eta(t-2), \ldots \eta(t-n))+\Delta \rho_{0}(t)}{\lambda_{j}}$

Where, $H$ is predictive functional, which can be realized by a moving window, and $H_{\text {round }}$ represents the rounding function.

\subsection{DETAILED PART OF THE PROPOSED METHOD}

We can constrain the error in a small range (less than three cycles) by formula (21), and we will detect and repair it detailly in this section. Even if we constrain the CS in a small range, it will affect the 


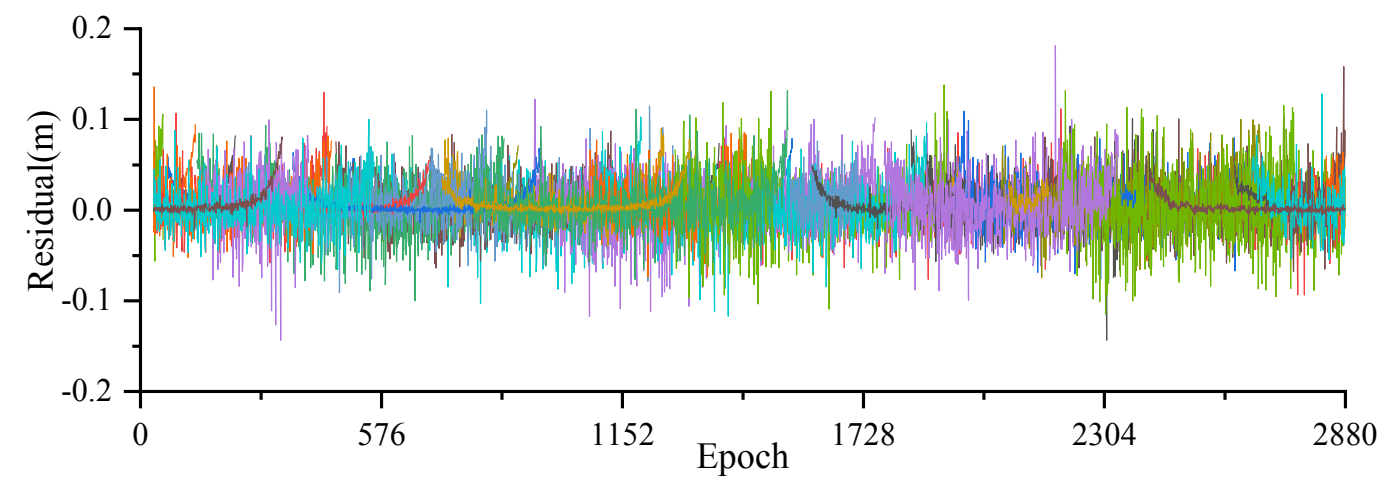

Fig. 2 The comparison of $\eta$ predicted and observed with low sampling rate data at a quiet ionospheric activity condition.

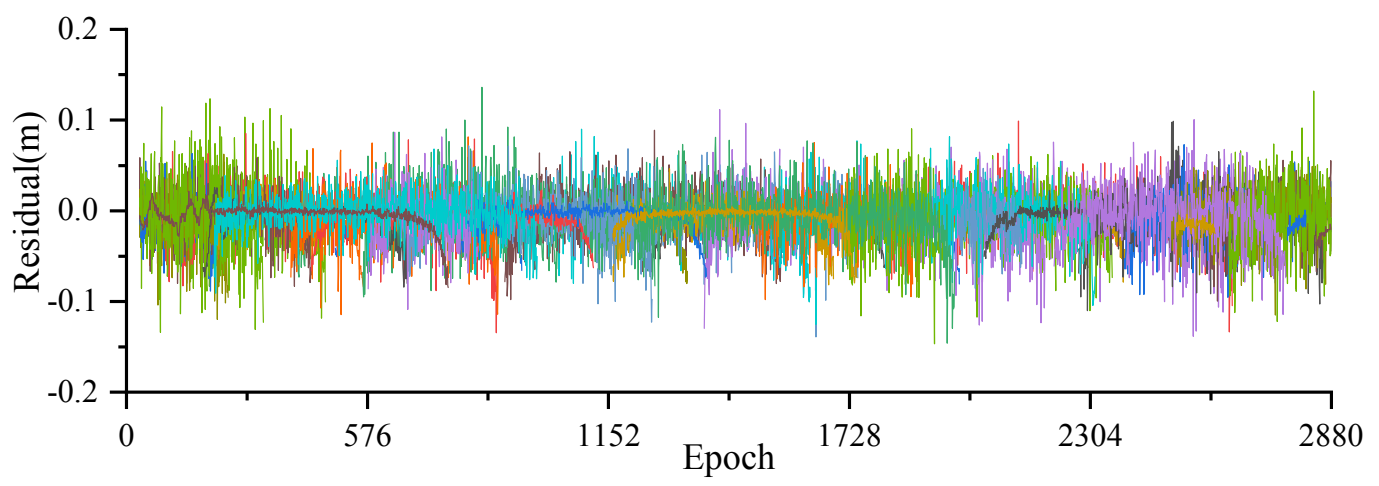

Fig. 3 The comparison of $\eta$ predicted and observed with low sampling rate data at a disturbed ionospheric activity condition.

smoothed pseudorange, ionospheric variation, dispersion, and non-dispersion. The influence on ionospheric variations is as follows:

$$
\begin{aligned}
\mu_{j} \Delta v & =\mu_{j}\left(\Delta \varphi_{1} \lambda_{1}-\Delta \varphi_{2} \lambda_{2}\right) /\left(\mu_{1}-\mu_{2}\right) \\
& =\mu_{j} \Delta v^{\prime}+\mu_{j}\left(\Delta z_{1} \lambda_{1}-\Delta z_{2} \lambda_{2}\right) /\left(\mu_{1}-\mu_{2}\right)
\end{aligned}
$$

In the above formula, $\mu_{j} \Delta v$ is real ionospheric variations while $\mu_{j} \Delta v^{\prime}$ is the influenced ionospheric variations. $\Delta z_{1}$ and $\Delta z_{2}$ represent the unique error on L1 and L2 frequency, respectively. We can present the smoothed pseudorange considering ionospheric changes and the error as follows:

$$
\begin{aligned}
P_{s j}^{\prime}(t) & =\frac{1}{\alpha} P_{j}(t)+\frac{\alpha-1}{\alpha}\left(P_{s j}^{\prime}(t-1)+\lambda_{j}\left(\varphi_{j}(t)-\right.\right. \\
& \left.\left.-\varphi_{j}(t-1)+\Delta z_{j}\right)-2 u_{j} \Delta v(t, t-1)\right)= \\
& =\frac{1}{\alpha} P_{j}(t)+\frac{\alpha-1}{\alpha}\left(P_{s j}^{\prime}(t-1)+\lambda_{j}\left(\varphi_{j}(t)-\right.\right. \\
& \left.\left.-\varphi_{j}(t-1)\right)-2 u_{j} \Delta v(t, t-1)\right)+ \\
& +\frac{\alpha-1}{\alpha}\left(\lambda_{j} \Delta z_{j}-\frac{2 \mu_{j}\left(\lambda_{1} \Delta z_{1}-\lambda_{2} \Delta z_{2}\right)}{\left(\mu_{1}-\mu_{2}\right)}\right)
\end{aligned}
$$

The influenced smoothed pseudorange considering the ionosphere variation is as follow:

$$
\Delta S=\frac{\alpha-1}{\alpha}\left(\Delta z_{j} \lambda_{j}+\frac{\mu_{j}\left(\Delta z_{1} \lambda_{1}-\Delta z_{2} \lambda_{2}\right)}{\left(\mu_{1}-\mu_{2}\right)}\right)
$$

Next, we construct four detectors, such as the ambiguity of MW-WL, L1 and L2, the residual of carrier phase and pseudorange. For simplicity of formula expression, it will not distinguish the smoothed pseudorange ignoring and considering ionospheric variation, and we take account of the ionospheric change in the following sections.

The first and second detector is the ambiguity variation of L1 and L2. The unaffected ambiguity of current epoch is equal to previous for L1 and L2, and we set a smaller threshold because of the higher precision of smoothed pseudorange. We design the detector as follows, and the threshold of those detectors is 1.5:

$$
E\left(\left(P_{s}(t)-\varphi(t)\right) / \lambda\right)=E\left(\left(P_{s}(t-1)-\varphi(t-1)\right) / \lambda\right)
$$

We make the GODN station as an example here, and show the TD model of L1 and L2 ambiguity calculated by smoothed pseudorange in Figure 4 and Figure 5. 


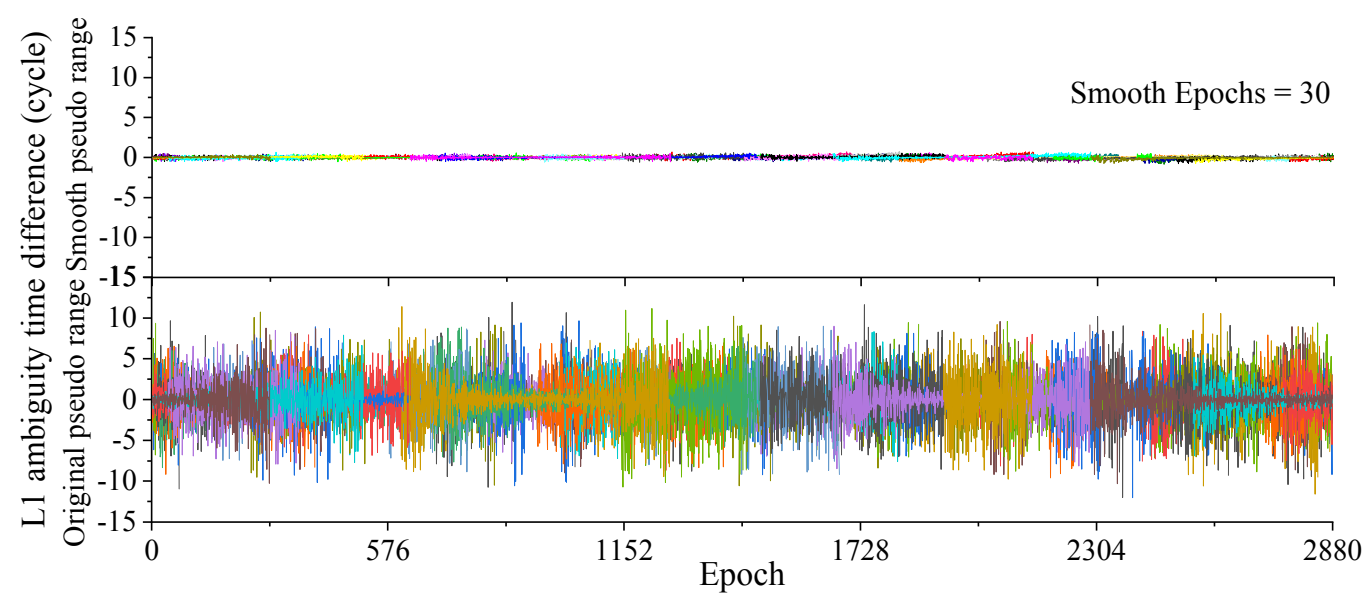

Fig. 4 The TD of L1 ambiguity.

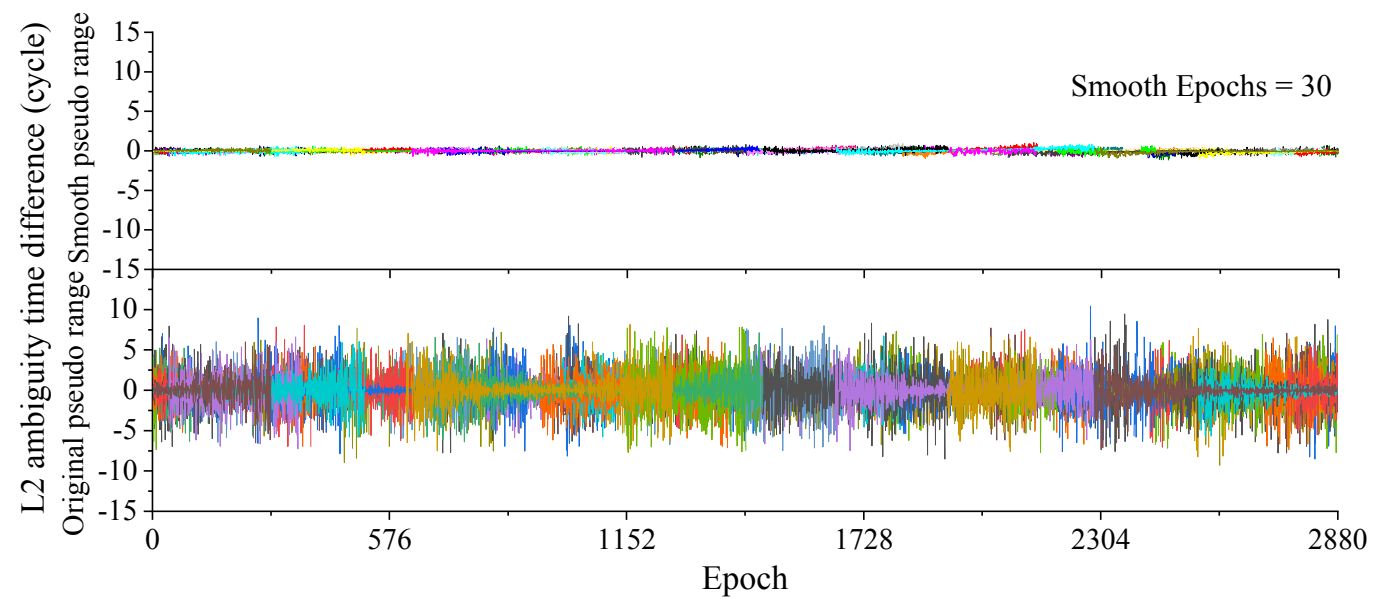

Fig. 5 The TD of L2 ambiguity.

From the above experimental and statistical results of GODN station, we can get that, on counting the difference TD model of integer ambiguity, the maximum is 11.94 cycles, and the minimum is -12.04 from original pseudorange while the smoothed is 0.75 and -0.89 cycles at L1 frequency. The peak is 8.16 cycles, and the bottle is -8.26 from original pseudorange while the smoothed is 0.57 and -0.68 cycles at L2. Thus, we can get a rough conclusion as follows, the original pseudorange of L1 can restrict the ambiguity within about 12 cycles while constraining the L2 frequency within about 8 cycles. Also, the smoothed pseudorange of L1 can limit the ambiguity within about 0.9 cycles while limiting the L2 signal within about 0.7 cycles. Therefore, we can design detectors by the difference TD model of integer ambiguity from L1 and L2 frequency.

We design the third detector by the TD model of the MW-WL and express it as follow, and the threshold of those detectors is 0.6 :

$\Delta z_{\Delta}=\left(\Delta z_{1}-\Delta z_{2}\right)=\frac{c\left(f_{1} \Delta P_{1}+f_{2} \Delta P_{2}\right)}{\left(f_{1}+f_{2}\right)\left(f_{1}-f_{2}\right)}-\left(\Delta \varphi_{1}-\Delta \varphi_{2}\right)$
Similarly, the method of L1 and L2 ambiguity variation is used to explore MW-WL ambiguity variation. Here we still take GODN station observations as an example, and make a TD model of MW-WL ambiguity, then shows the result in Figure 6.

From the above experimental and statistical results of GODN station, we can get that, on counting the difference TD model of MW-WL integer ambiguity obtained, the maximum is 2.87 cycles, and the minimum is -2.82 from original pseudorange while the smoothed is 0.07 and -0.09 cycles. Therefore, we can design detectors by the difference TD model of MW-WL ambiguity.

The fourth detector is residual of pseudorange and phase observation, and the form is as follows, and the threshold of those detectors is 0.15 :

$$
\begin{aligned}
& E\left(\Delta P_{1}-\Delta P_{2}\right)=E\left(\Delta \varphi_{1} \lambda_{1}-\Delta \varphi_{2} \lambda_{2}\right) \\
& E\left(\left(\Delta P_{1}-\Delta P_{2}\right)-\left(\Delta \varphi_{1} \lambda_{1}-\Delta \varphi_{2} \lambda_{2}\right)\right)=0
\end{aligned}
$$

Like the detector designed by MW-WL ambiguity variation, the method of L1 and L2 ambiguity variation is used to explore residual of pseudorange and phase observation. Here we still take 


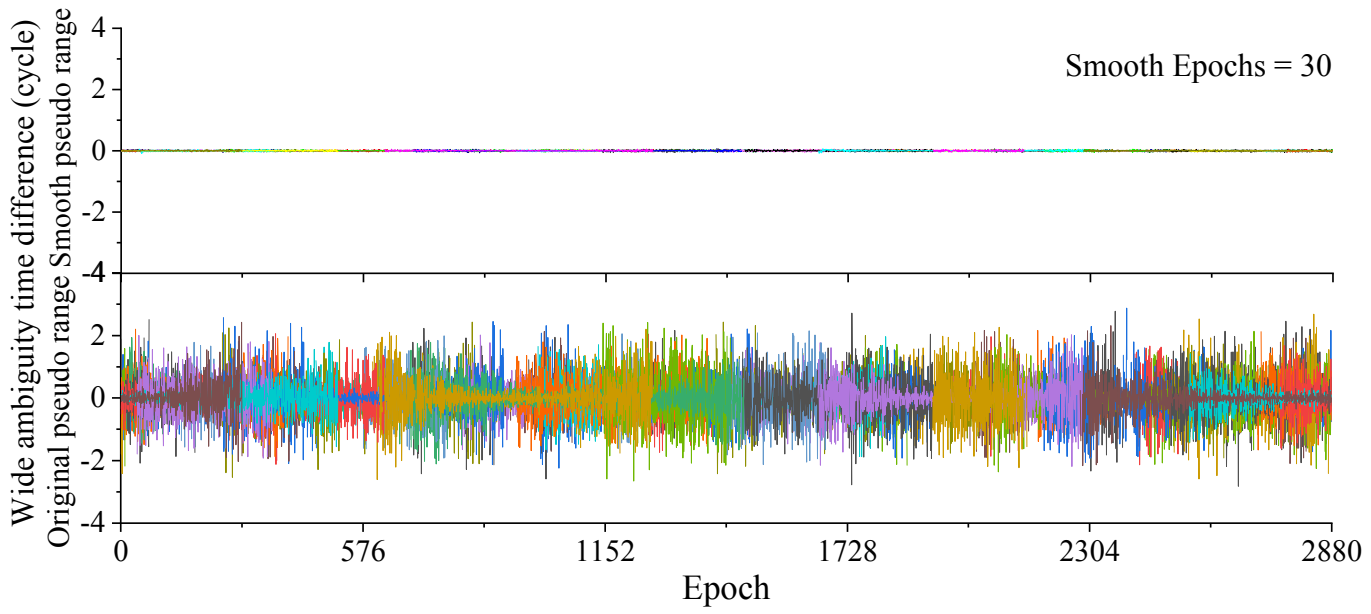

Fig. 6 The TD of MW-WL ambiguity.

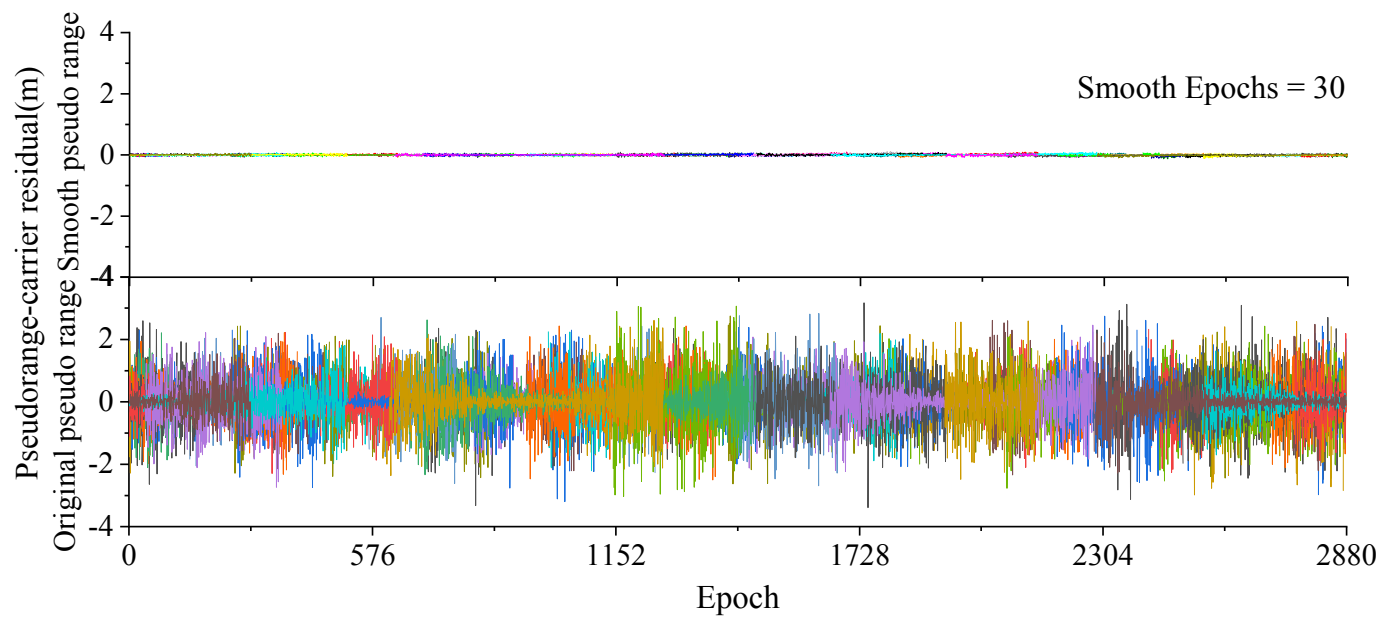

Fig. 7 TD residual between pseudorange and carrier residual.

GODN station as an example, and make a difference of pseudorange and phase observation, and show the result in Figure 7.

From the above experimental and statistical results of GODN station, we can get that, for the residual of original pseudorange and carrier phase observation, the maximum and minimum is $3.17 \mathrm{~m}$ and $-3.39 \mathrm{~m}$. Correspondingly, for the residual of smoothed pseudorange and carrier phase observation, the maximum and minimum are only $0.11 \mathrm{~m}$ and $0.13 \mathrm{~m}$.

There are a few particular CS can pass the above test, and the next verification is the uniformity of prior and posterior ionospheric variation estimated by LS, and the threshold of those detectors is 0.02 :

$E\left(\Delta v_{p r}\right)=E\left(\Delta v_{p o}\right)$

Where $\Delta v_{p r}$ and $\Delta v_{p o}$ indicate prior and posterior ionospheric variation, respectively.

We design a detector by the difference of prior and posterior ionospheric variation and the minimum sum of the residual square. Because of the high precision of smoothed pseudorange, the difference between the two ionospheric variations is quite a suitable detector.

For the CS is an error with integer characteristics, so the hypothesis test can be used to detect and repair it. To reduce the number of parameters, the null hypotheses hold that there is no error while the alternative assumes there exist, and we describe the hypotheses as follow:

$$
\begin{aligned}
& H_{0}: \Delta z=0 \\
& H_{a}: \Delta z \neq 0
\end{aligned}
$$

If the null hypothesis $H_{0}$ is correct, we can get that:

$\left\{\begin{array}{l}E\left\{\Delta P_{s}\right\}=\Delta \rho-u \Delta v \\ E\{\lambda \Delta \varphi\}=\Delta \rho+u \Delta v \Rightarrow E\{\mathrm{y}\}=A x \\ E\{\Delta \dot{v}\}=\Delta v\end{array}\right.$

Where $\Delta P_{s}=\left[\Delta P_{s 1}, \Delta P_{s 2}, \cdots, \Delta P_{s f}\right]$, $\Delta \varphi=\left[\Delta \varphi_{1}, \Delta \varphi_{2}, \cdots, \Delta \varphi_{f}\right]$ and $\Delta \dot{v}$ is the predicted ionospheric variation by previous epochs. 


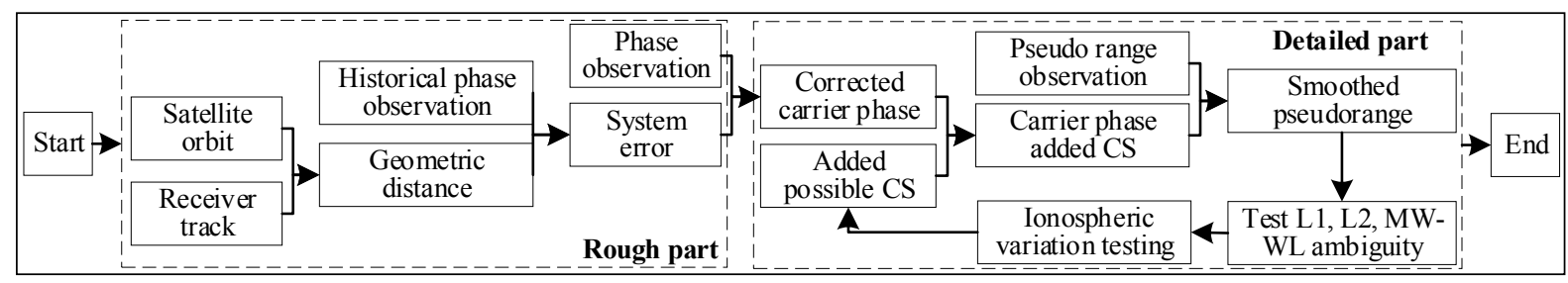

Fig. 8 Flow chart of the proposed method.

If the null hypothesis is correct, there are $2 f+1$ observations and only 2 parameters to be estimated in the equation (31). Relying on the parameter estimation model, we should pay attention to that it has quite a strength model because its freedom is $2 f-1$. Thus, any CS will make a disturbance on $y=A x$, which can lead to a deviation of parameter estimation.

\subsection{THE WHOLE PROCESS OF THE PROPOSED CSDR METHOD}

In this section, we introduced the entire process of the proposed algorithm by flow chart and explanation language, and show the flow chart in Figure 8 .

From Figure 8, we can realize the proposed method by the following steps:

1. Calculate systematic error. We obtain the geometric distance from satellite orbit and receiver track and make a TD model for geometric distance and phase observations. Then the TD model difference between satellite-toreceiver geometric distance and phase observations of the current epoch is predicted by the historical information.

2. Detect and repair the massive error. Then the difference of current epoch is also estimated by satellite-to-receiver geometric distance and phase observation. The two difference is compared to detect and fix the massive error.

3. Construct detectors. We calculate the ionospheric variation, and smooth pseudorange considering the ionospheric variety. Next, we establish detectors by the ambiguity of MW-WL, L1 and L2, residual of carrier phase and smoothed pseudorange, respectively.

4. Detect and repair the small error. We search the unique error by adding error from -3 to +3 cycles to original phase observation and verify the detectors established at step (3).

5. Detect and repair some value not handled at step (4). The ionospheric variation predicted and observed is used as a detector for the specific value.

Notes: in order to eliminate the influence of prior unit weight variance on parameter estimation, the iterative LS is used to get the minimum difference of posterior and prior unit weight variance. Also, the ultra-fast precision ephemeris downloaded in advance, or the navigation file is used to get satellite orbit.
Moreover, it assumed that there is no error or has repaired at previous epochs for establishing detectors.

\section{EXPERIMENTAL RESULTS AND ANALYSIS 4.1. DATA DESCRIPTION}

To verify the applicability of the proposed method, the real measured datasets from the IGS with low sampling rate collected on October 27, 2018, are used to verify the proposed method. We make a distribution of these selected stations in Figure 9, and the datasets have good representativeness and diversities. From the figure, we can get that the station's distribution is all over the world. From the east to the west direction, there are 3 stations in the eastern hemisphere, 5 stations in the western, and 1 station nearby the boundary. From the east to the west direction, there are 2 in the southern hemisphere while 7 in the northern hemisphere. Furthermore, the datasets from inland and littoral are both selected in the datasets, and we test receivers and antennas from different manufacturers and brands, such as Trimble, Javad, Septentro, Tps.

\subsection{THE SIMULATION CS AND RESULT}

The receiver position can obtain from SPP by the observations of the several beginning epochs firstly when we used the proposed method. For a static receiver, the receiver position is a constant value, and receiver coordinates from SPP can promise enough precision calculating the geometric distance of satellite-to-receiver. We use a moving smoothing window to calculate the systematic error while using a polynomial fitting to predict the ionospheric variation.

To verify the algorithm's efficiency, we add artificial error from -3 to 3 cycles at both frequencies for all epochs except for the several beginning, since we establish the detectors by the several beginning epochs (30 epochs). A particular typical combination, $(-1,-1)$, is added since it is insensitive to detectors designed by MW-WL. Moreover, a more special combination, $(-77,-60)$ and $(-9,-7)$, are added, which have an equal ratio with frequencies. Moreover, the added error can make a representative of the most insensitive error. To obtain a more eloquent result, all epochs, except for the several bargaining epochs, are simulated and tested in this study while only part of epochs at other researches. The massive error can be limited to a small range (less than 3 cycles) by the 


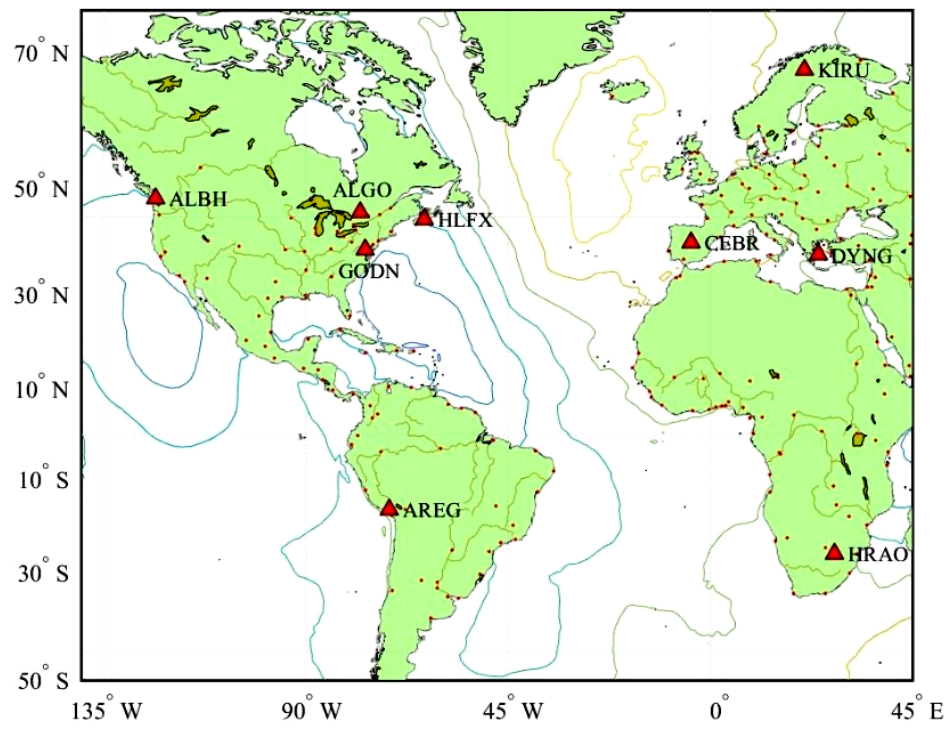

Fig. 9 The distribution of the test stations.

Table 1 The CSDR statistical information of observations with a low sampling rate (30 s).

\begin{tabular}{|c|c|c|c|c|c|c|c|}
\hline Station & EN & AVS & Frequency & ACSE & Correct (Rate) & Error (Rate) & Failure (Rate) \\
\hline \multirow{2}{*}{ ALBH } & \multirow{2}{*}{25,030} & \multirow{2}{*}{8.69} & $\mathrm{~L}_{1}$ & 23,286 & $23,198(99.62 \%)$ & $62(0.27 \%)$ & $26(0.11 \%)$ \\
\hline & & & $\mathrm{L}_{2}$ & 23,286 & $23,198(99.62 \%)$ & $62(0.27 \%)$ & $26(0.11 \%)$ \\
\hline \multirow{2}{*}{ ALGO } & \multirow{2}{*}{24,915} & \multirow{2}{*}{8.65} & $\mathrm{~L}_{1}$ & 23,207 & $23,058(99.36 \%)$ & $138(0.59 \%)$ & $11(0.05 \%)$ \\
\hline & & & $\mathrm{L}_{2}$ & 23,207 & $23,058(99.36 \%)$ & $138(0.59 \%)$ & $11(0.05 \%)$ \\
\hline \multirow{2}{*}{ AREG } & \multirow{2}{*}{26,190} & \multirow{2}{*}{9.09} & $\mathrm{~L}_{1}$ & 24,080 & $23,994(99.64 \%)$ & $77(0.32 \%)$ & $9(0.04 \%)$ \\
\hline & & & $\mathrm{L}_{2}$ & 24,080 & $23,994(99.64 \%)$ & $77(0.32 \%)$ & $9(0.04 \%)$ \\
\hline \multirow{2}{*}{ CEBR } & \multirow{2}{*}{24,251} & \multirow{2}{*}{8.42} & $\mathrm{~L}_{1}$ & 22,708 & $22,606(99.55 \%)$ & $82(0.36 \%)$ & $20(0.09 \%)$ \\
\hline & & & $\mathrm{L}_{2}$ & 22,708 & $22,606(99.55 \%)$ & $82(0.36 \%)$ & $20(0.09 \%)$ \\
\hline \multirow{2}{*}{ DYNG } & \multirow{2}{*}{24,531} & \multirow{2}{*}{8.52} & $\mathrm{~L}_{1}$ & 22,507 & $22,326(99.20 \%)$ & $174(0.77 \%)$ & $7(0.03 \%)$ \\
\hline & & & $\mathrm{L}_{2}$ & 22,507 & $22,330(99.21 \%)$ & $166(0.74 \%)$ & $11(0.05 \%)$ \\
\hline \multirow{2}{*}{ GODN } & \multirow{2}{*}{24,777} & \multirow{2}{*}{8.60} & $\mathrm{~L}_{1}$ & 23,059 & $23,022(99.84 \%)$ & $28(0.12 \%)$ & $9(0.04 \%)$ \\
\hline & & & $\mathrm{L}_{2}$ & 23,059 & $23,022(99.84 \%)$ & $28(0.12 \%)$ & $9(0.04 \%)$ \\
\hline \multirow{2}{*}{ HLFX } & \multirow{2}{*}{24,472} & \multirow{2}{*}{8.50} & $\mathrm{~L}_{1}$ & 22,773 & $22,652(99.47 \%)$ & $98(0.43 \%)$ & $23(0.10 \%)$ \\
\hline & & & $\mathrm{L}_{2}$ & 22,773 & $22,652(99.47 \%)$ & $98(0.43 \%)$ & $23(0.10 \%)$ \\
\hline \multirow{2}{*}{ HRAO } & \multirow{2}{*}{24,070} & \multirow{2}{*}{8.36} & $\mathrm{~L}_{1}$ & 22,302 & $22,263(99.83 \%)$ & $32(0.14 \%)$ & $7(0.03 \%)$ \\
\hline & & & $\mathrm{L}_{2}$ & 22,302 & $22,263(99.83 \%)$ & $32(0.14 \%)$ & $7(0.03 \%)$ \\
\hline \multirow{2}{*}{ KIRU } & \multirow{2}{*}{28,901} & \multirow{2}{*}{10.04} & $\mathrm{~L}_{1}$ & 26,702 & $26,509(99.28 \%)$ & $176(0.66 \%)$ & $17(0.06 \%)$ \\
\hline & & & $\mathrm{L}_{2}$ & 26,702 & $26,507(99.27 \%)$ & $174(0.65 \%)$ & $21(0.08 \%)$ \\
\hline
\end{tabular}

Note: EN means Epoch number. AVS means average visible satellites. ACSE means add CS epoch. The error means one of the combinations can pass the test, but it is not the real error while failure means all of the combinations cannot pass the test, even if we added the real unique error.

rough step, so the massive error is counted according to the small when counting statistical information. We show the statistical information in Table 1.

There are only 31 GPS satellites in the sky because the G04 is out of service on the observation day. According to statistics information in Table 1, the maximum of average visible satellites is KIRU station, which is 10.04 with 57,802 observations in all, while the minimum is HRAO station, which is 8.36 with 48,140 observations in all. On counting the information of CSDR, the lowest success rate station is DYNG with $99.20 \%$ on the L1 frequency and $99.21 \%$ on L2, and the highest success rate station is HRAO with $99.83 \%$ on both L1 and L2. The results show that the success rate is over $99.2 \%$ for all station, which verifies the validity and reliability of the proposed method. On counting the failure rate, the highest failure rate station is ALBH with $0.11 \%$ on both L1 and L2, and there are only 52 observations cannot execute the proposed method. The lowest failure rate station is HRAO with $0.03 \%$ on both L1 and L2 frequency, and there are only 14 observations cannot execute the proposed method. From the above statistical results, the situation of CSDR has a significant similarity between $\mathrm{L} 1$ and L2, which indicates that the failure and succeed result of L1 and L2 would affect and restrict each other. 


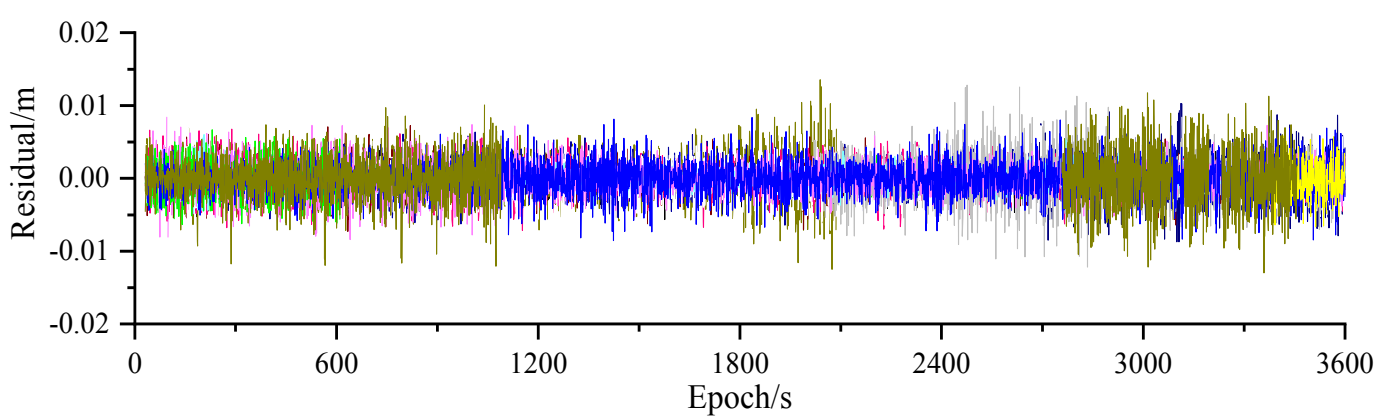

Fig. 10 The comparison of $\eta$ by predicted and observed with high sampling rate data.

Table 2 The CSDR statistical information of dataset with a high sampling rate $(1 \mathrm{~s})$.

\begin{tabular}{|c|c|c|c|c|c|c|c|c|}
\hline Station & EN & AVS & Frequency & ACSE & Correct & Correct Rate & Failure & Failure Rate \\
\hline \multirow{2}{*}{ ALBH } & \multirow{2}{*}{31,725} & \multirow{2}{*}{8.81} & $\mathrm{~L}_{1}$ & 31,243 & 31,243 & $100.00 \%$ & 0 & $0.00 \%$ \\
\hline & & & $\mathrm{L}_{2}$ & 31,243 & 31,243 & $100.00 \%$ & 0 & $0.00 \%$ \\
\hline \multirow{2}{*}{ ALGO } & \multirow{2}{*}{34,756} & \multirow{2}{*}{9.65} & $\mathrm{~L}_{1}$ & 34,292 & 34,292 & $100.00 \%$ & 0 & $0.00 \%$ \\
\hline & & & $\mathrm{L}_{2}$ & 34,292 & 34,292 & $100.00 \%$ & 0 & $0.00 \%$ \\
\hline \multirow{2}{*}{ AREG } & \multirow{2}{*}{34,080} & \multirow{2}{*}{9.47} & $\mathrm{~L}_{1}$ & 33,709 & 33,709 & $100.00 \%$ & 0 & $0.00 \%$ \\
\hline & & & $\mathrm{L}_{2}$ & 33,709 & 33,709 & $100.00 \%$ & 0 & $0.00 \%$ \\
\hline \multirow{2}{*}{ CEBR } & \multirow{2}{*}{41,247} & \multirow{2}{*}{11.46} & $\mathrm{~L}_{1}$ & 40,576 & 40,576 & $100.00 \%$ & 0 & $0.00 \%$ \\
\hline & & & $\mathrm{L}_{2}$ & 40,576 & 40,576 & $100.00 \%$ & 0 & $0.00 \%$ \\
\hline \multirow{2}{*}{ DYNG } & \multirow{2}{*}{44,248} & \multirow{2}{*}{12.29} & $\mathrm{~L}_{1}$ & 42,813 & 42,813 & $100.00 \%$ & 0 & $0.00 \%$ \\
\hline & & & $\mathrm{L}_{2}$ & 42,813 & 42,813 & $100.00 \%$ & 0 & $0.00 \%$ \\
\hline \multirow{2}{*}{ GODN } & \multirow{2}{*}{35,931} & \multirow{2}{*}{9.98} & $\mathrm{~L}_{1}$ & 34,917 & 34,915 & $99.99 \%$ & 2 & $0.01 \%$ \\
\hline & & & $\mathrm{L}_{2}$ & 34,917 & 34,915 & $99.99 \%$ & 2 & $0.01 \%$ \\
\hline \multirow{2}{*}{ HLFX } & \multirow{2}{*}{34,115} & \multirow{2}{*}{9.48} & $\mathrm{~L}_{1}$ & 33,685 & 33,685 & $100.00 \%$ & 0 & $0.00 \%$ \\
\hline & & & $\mathrm{L}_{2}$ & 33,685 & 33,685 & $100.00 \%$ & 0 & $0.00 \%$ \\
\hline \multirow{2}{*}{ HRAO } & \multirow{2}{*}{27,854} & \multirow{2}{*}{7.74} & $\mathrm{~L}_{1}$ & 27,262 & 27,262 & $100.00 \%$ & 0 & $0.00 \%$ \\
\hline & & & $\mathrm{L}_{2}$ & 27,262 & 27,262 & $100.00 \%$ & 0 & $0.00 \%$ \\
\hline \multirow{2}{*}{ KIRU } & \multirow{2}{*}{42,551} & \multirow{2}{*}{11.82} & $\mathrm{~L}_{1}$ & 41,892 & 41,892 & $100.00 \%$ & 0 & $0.00 \%$ \\
\hline & & & $\mathrm{L}_{2}$ & 41,892 & 41,892 & $100.00 \%$ & 0 & $0.00 \%$ \\
\hline
\end{tabular}

Different method is suitable for low and high sampling rate dataset as the different property. For low sampling rate data, the non-dispersion error of adjacent epochs is too large to predict, so the predicted $\eta$ by polynomial fitting can constraint the error into a small range. However, for high sampling rate data, the non-dispersion error of adjacent epochs can be predicted accurately, so the $\eta$ predicted is a good value for identifying the error. Similar to low sampling rate data, we make an example for CEBR station data collecting from AM 9:00 to 10:00 on 27 October 2018, and show the $\eta$ difference from prediction and observation in Figure 10.

From the figure, the $\eta$ difference from the two is quite a good value, although there is some difference among different satellites. However, the $\eta$ residual of low sampling rate data may be an immense value, and the result is in Figures 1-3.

Correspondingly, the corresponding dataset with high sampling rate is used to verify the proposed method. Except for the several beginning epochs, we test all epochs of both frequencies and show the statistical information in Table 2.
According to the statistics information in Table 2, when counting implementation rate, the highest station is AREG whose proportion is $98.91 \%$ with 67,418 executed in all 68,160 observation, while the lowest is DYNG whose proportion is $96.76 \%$ with 88,496 implemented in all 85,626 observations, among the test datasets. When counting the average number of visible satellites, the maximum is KIRU, which is 12.29 with 88,496 observations in all, while the minimum is HRAO, which is 7.74 with a total of 55,708 observations. When counting the information of CSDR, there are only 4 observations is a failure which station is GODN, in all test station with a total of 320,389 observations. Thus, for high sampling rate data, the $\eta$ of current epoch can be predicted accurately, and the proposed method is sufficient for a static receiver.

\subsection{ANALYSIS AND SUMMARY OF CYCLE SLIP DETECTION AND REPAIR RESULT}

By analyzing the epochs which are wrong and failed to detect and repair $\mathrm{CS}$, and the following reasons can account for this phenomenon.

The first category reason is the precision of ephemeris and the interpolating method, which mainly 
affect the rough step of the proposed method for both low and high sampling rate data. By polynomial fitting methods, the precision beginning and ending epochs will reduce rapidly, and as much ephemeris data as possible should be used to improve orbit accuracy. Simultaneously, the TD model eliminates the systematic error while enlarges the random error, so the systematic error makes no influence on the result while the result can be affected by the random error.

The second category reason is the gross error on the original pseudorange, which will lead a mistake on the smoothed pseudorange. Furthermore, it leads that the ambiguity of MW-WL, L1, L2, and the residual error of smoothed pseudorange and phase observation will deviate from the real value, and the result of LS is unreliable.

The third category reason is the prediction method and the over-draft of the non-dispersion, which can affect the first process of the proposed method for both low and high sampling rate data. The inaccurate prediction of the non-dispersion causes the 2 epochs failure of the high sampling rate data. Additionally, the erroneous prediction of the nondispersion is unable to lock the CS at a small range (less than 3 cycles) for the low sampling rate.

The fourth category reason is the inaccurate prediction of the ionosphere. Even if the error has repaired, the detector is unable to pass the test for the inaccurate prediction of the ionosphere.

\section{CONCLUSION}

We use different methods for different sampling rate data in this study. For high sampling rate data, double constraints from systematic error and satellite orbit are enough. The experimental result shows excellent reliability since there are only 2 epochs are failed among 640,778 epochs by the rough part when testing datasets with a high sampling rate.

A step method based on double-constraint of ephemeris and smoothed pseudorange is suitable for low sampling rate data. The massive error is constrained in a small range by systematic error and satellite orbit at the first process, and we add -3 to +3 integer error on original phase observation and smooth the pseudorange. Then the ambiguity of MWWL, L1, L2 is tested to decide whether the added CS is correct. Furthermore, the method uses the difference between prior and posterior ionosphere variation to identify the error. The experimental result shows high reliability and validity for the dataset with a low sampling rate because the lowest succeed rate is 99.20 $\%$ in 9 test stations observations dataset.

We only test the GPS datasets for validating the proposed method in this study because the computation increases multiply with the increasing of the systems. In the future, we will design more detectors to narrow the searching space, and use multi-GNSS to validate the detectors such as GLONASS (Global Navigation Satellite System),
BDS (BeiDou Navigation Satellite System), GALILEO satellite navigation system, QZSS (QuasiZenith Satellite System) and IRNSS (Indian Regional Navigation Satellite System). Meanwhile, the dynamic information of the receiver will cause an incorrect calculation of the satellites-to-receiver, so only the static receiver is studied in this paper. In the future, the INS information will facilitate and assist error detection and repair for a dynamic receiver for further development.

\section{ACKNOWLEDGMENT}

The work is sponsored by the National Natural Science Foundation of China (41674008, 41604006, 41874006, 41804029) and Postgraduate Research \& Practice Innovation Program of Jiangsu Province (KYCX19_2161).

\section{REFERENCES}

Altmayer, C.: 2000, Enhancing the integrity of integrated GPS/INS systems by cycle slip detection and correction. IEEE Intelligent Vehicles Symposium 2000 (Cat. No.00TH8511), Dearborn, Michigan, USA, 5-5 October, 174-179.

DOI: $10.1109 /$ IVS.2000.898337

Banville, S. and Langley, R.B.: 2013, Mitigating the impact of ionospheric cycle slips in GNSS observations. J. Geod., 87, 2, 179-193.

DOI: $10.1007 / \mathrm{s} 00190-012-0604-1$

Blewitt, G.: 1990, An automatic editing algorithm for GPS data. Geophys. Res. Lett., 17, 3, 199-202. DOI: 10.1029/GL017i003p00199

Cai, C., Liu, Z., Xia, P. and Dai, W.: 2013, Cycle slip detection and repair for undifferenced GPS observations under high ionospheric activity. GPS Solut., 17, 2, 247-260. DOI: $10.1007 / \mathrm{s} 10291-012-0275-7$

Colombo, O.L., Bhapkar, U.V. and Evans, A.G.: 1999, Inertial-aided cycle-slip detection/correction for precise, long-baseline kinematic GPS. Proceedings of the 12th International Technical Meeting of the Satellite Division of The Institute of Navigation (ION GPS 1999), Nashville, Tennessee, USA, 14-17 September, 1915-1922.

de Lacy, M.C., Reguzzoni, M. and Sansò, F.: 2012, Realtime cycle slip detection in triple-frequency GNSS. GPS Solut., 16, 3, 353-362. DOI: $10.1007 / \mathrm{s} 10291-011-0237-5$

de Lacy, M.C., Reguzzoni, M., Sansò, F. and Venuti, G.: 2008, The Bayesian detection of discontinuities in a polynomial regression and its application to the cycleslip problem. J. Geod., 82, 9, 527-542. DOI: $10.1007 / \mathrm{s} 00190-007-0203-8$

Du, S. and Gao, Y.: 2012, Inertial aided cycle slip detection and identification for integrated PPP GPS and INS. Sensors, 12, 11, 14344-14362. DOI: $10.3390 / \mathrm{s} 121114344$.

Hatch, R.R.: 1982, The synergism of GPS code and carrier measurements. 3rd International Geodetic Symposium on Satellite Doppler Positioning, Physical Science of New Mexico State University, New Mexico, USA, 0812 February, 1213-1231.

Hieu, L.V., Ferreira, V.G., He, X. and Xu, T.: 2014, Study on cycle-slip detection and repair methods for a single 
dual-frequency global positioning system (GPS) receiver. Boletim De Ciencias Geodesicas, 20, 4, 9841004. DOI: 10.1590/S1982-21702014000400054

Huang, L., Song, L. and Wang, Y.: 2012, BeiDou triplefrequency geometry-free phase combination for cycleslip detection and correction. Acta Geod. Cartogr. Sin., 41, 5, 763-768.

DOI: $10.1007 / \mathrm{s} 11783-011-0280-\mathrm{z}$

Lee, H., Wang, J. and Rizos, C.: 2003, Effective cycle slip detection and identification for high precision GPS/INS integrated systems. J. Navigation, 56, 3, 475-486. DOI: 10.1017/S0373463303002443

Li, B., Qin, Y. and Liu, T.: 2019, Geometry-based cycle slip and data gap repair for multi-GNSS and multifrequency observations. J. Geod., 93, 3, 399-417. DOI: $10.1007 / \mathrm{s} 00190-018-1168-5$

Li, J., Yang, Y., Xu, J. and He, H.: 2011, Real-time cycleslip detection and repair based on code-phase combinations for GNSS triple-frequency undifferenced observations. Acta Geod. Cartogr. Sin., 40, 6, 716-717. DOI: 10.1007/s11769-011-0446-4

Li, Z., Gao, J. and Wang, J.: 2016, Inertial aided cycle slip detection and repair for PPP/INS tightly coupled navigation. J. Navigation, 69, 6, 1357-1378. DOI: $10.1017 / \mathrm{s} 0373463316000023$

Liu, Z.: 2011, A new automated cycle slip detection and repair method for a single dual-frequency GPS receiver. J. Geod., 85, 3, 171-183.

DOI: $10.1007 / \mathrm{s} 00190-010-0426-y$

Melbourne, W.G.: 1985, The case for ranging in GPS based geodetic systems. First International Symposium on Precise Positioning with the Global Positioning System, Department of Commerce, Rockville, Maryland, USA, 15-19 April, 373-386.

Serrano, L., Kim, D., Langley, R.B., Itani, K. and Ueno, M.: 2004, A GPS velocity sensor: How accurate can it be? - A first look. Ion National Technical Meeting, the Catamaran Resort Hotel, San Diego, California, USA, 26-28 January, 875-885.

Wübbena, G.: 1985, Software developments for geodetic positioning with GPS using TI-4100 code and carrier measurements, First International Symposium on Precise Positioning with the Global Positioning System, United States Department of Commerce, Rockville, Maryland, USA, 15-19 April, 403-412.
Xiao, G., Mayer, M., Heck, B., Sui, L., Zeng, T. and Zhao, D.: 2018, Improved time-differenced cycle slip detect and repair for GNSS undifferenced observations. GPS Solut., 22, 1, 1-13. DOI: 10.1007/s10291-017-0677-7

Younsil, K., Junesol, S., Ho, Y., Byungwoon, P. and Changdon, K.: 2013, GPS cycle-slip detection with low-cost IMU and single-frequency receiver of land vehicle. Proceedings of the ION 2013 Pacific PNT Meeting, Marriott Waikiki Beach Resort \& Spa, Honolulu, Hawaii, USA, 23-25 April, 1032-1039. DOI: $10.1117 / 12.2028852$

Zhang, X. and Li, P.: 2016, Benefits of the third frequency signal on cycle slip correction. GPS Solut., 20, 3, 451460. DOI: $10.1007 / \mathrm{s} 10291-015-0456-2$.

Zhang, X. and Li, X.: 2012, Instantaneous re-initialization in real-time kinematic PPP with cycle slip fixing. GPS Solut., 16, 3, 315-327. DOI: $10.1007 / \mathrm{s} 10291-011-0233-9$

Zhao, D., Roberts, G.W., Hancock, C.M., Lau, L. and Bai, R.: 2019, A triple-frequency cycle slip detection and correction method based on modified HMW combinations applied on GPS and BDS. GPS Solut., 23, 1, 22. DOI: 10.1007/s10291-018-0817-8. 